\title{
Seed Quality Analysis Using Image Processing and ANN
}

\author{
Miss Shivpriya Desai \\ M.E (Digital System), JSPM's JSCOE, \\ Pune, India
}

\author{
Dr. A. P. Rao \\ Assistant Professor, JSPM's JSCOE, \\ Pune, India
}

\begin{abstract}
The use of good quality seed is important for the satisfactory production of a good quality crop and is essential for export in markets. Quality control is very important in food industry because based on quality of food products are classified and graded into different grades. Quality of grain is evaluated manually but it is very much, time consuming, may be varying results and costly. Digital image processing can be used for quality evaluation and grading of agricultural food in industry. One of the major steps in quality analysis of grains is grading of grains, which is normally done either manually or using expensive electronics equipments. We propose a simple method based on image processing and using Artificial Neural Network for grading of rice granules Here grading will be performed by calculating rice seed major axis length ,miner axis length, area ,eccentricity and perimeter. Result will be displayed on LCD and also there is an audio message.
\end{abstract}

The paper presents a solution of grading and analysis of rice grains on the basis of grain size and shape using image processing techniques and artificial neural network. This method requires minimum time and it is low in cost.

Keywords: Grain quality, Rice, Image Processing and Analysis, ANN

\section{INTRODUCTION}

The demand for quality of food products we consume is increasing day by day. As the literacy rate is increasing in India so the need for quality of food products is increasing. India is the second largest producer of rice grains. As the production of rice is increasing so the demand for its quality is also increasing. Some traders discharge poor quality products to the shopkeepers. Because of such practices there are numerous low-grade quality grains increasing to the market place day by day. This demand for quality of food grains is increasing because some of the traders cheat the shopkeepers by selling poor quality food grains which contains foreign particles like stones, leaf, broken and damaged seeds etc. This kind of low quality of rice is sold without noticed even there is no special scheme to detect such poor quality grains. Therefore it is been a problem for both consumers and sellers.

As the technology is growing wider people are adopting the new technologies. They are not using the old techniques. The growth in technology is making people more demanding towards the things they use and consume, this is the reason why everything is becoming automated. The use of Image processing techniques for testing the quality of rice grains is inexpensive and is less time consuming also it gives accurate results.. The quality of grain is tested based on its color, size, shape and major axis length, minor axis length in this method.

Previously, biological methods and chemical methods can be used for the identification of rice grain varieties and quality. But these methods are very expensive and time consuming and results are not accurate. On the other hand the machine vision or the digital image processing is a non destructive method. It is a very fast and inexpensive process compared to the biological methods.

\section{LITERATURE REVIEW}

In this Literature survey number of author proposed their work and is what follows. Kaur and Verma, [2] have proposed computer vision techniques for grading of rice based on their sizes. They have done grading on the basis of medium ,long and short length rice. The images are acquired using a digital camera having high pixel resolution. The camera is located at a position normal to the object. 
The grading formula \& standards were acquired from the analysis procedure for grading rice followed in India.

Jagdeep Singh and Banga [3] have proposed image processing techniques for grading of rice samples based on their sizes. The images were captured using a Flat Bed Scanner (FBS) and even high resolution digital camera also be used. Image acquired is then converted to binary image and then apply morphological operations and by finding the properties of the connected components in the image the objects features were extracted. Maheshwari [4] proposed image processing techniques for identification of two varieties of rice based on their shape and size of grains. Sample grains spread on the black paper. Image will captured using a digital camera, the edge detection operation were performed to calculate the Geometric parameters. Based on different parameters they classified rice seeds into three parts namely normal rice, long rice and small rice seeds .Here they displayed the count of normal, long and small rice seeds on screen.

Harpreet Kaur and Baljit Singh have proposed a technique for classification and grading of rice grains using multi-class SVM. Harpreet and Baljit captured the images of sample by using black sheet of paper as background. The images were preprocessed to remove noise. The images then subdivided into its parts by using different image segmentation methods. The content of chalky grains were calculated, further the chalky degree is calculated. The length of the grain is analyzed the grain shape is defined based on its length and width. Based on this length the purity of the grain is calculated. If its length is greater than $75 \%$ than it's considered as unbroken of else it's taken as broken. Here an SVM that is support vector machine which is a new type of classifier is used.

Anami, and Savakar [8] have proposed a technique in identifying and elimination of foreign particles in the image samples. They used material other than food grain as foreign body. When they observed the foreign bodies present were stone, leaves, dust, other types of grains etc. Here they considered both colour and texture for their study. They carried out the work by first collecting the images of the rice samples. The features of the images were acquired and were stored as knowledge base. Texture features were extracted in order to recognize and classify the food grains.

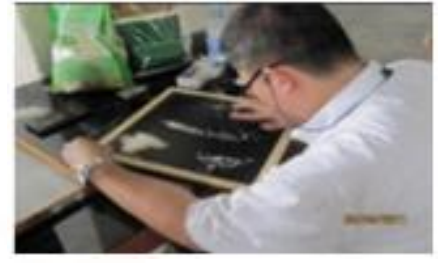

Fig 2: Physical inspection and identification of rice

Ajay [7] have proposed a quality evaluation of rice grains using morphological methods. Physical features play a vital role in classification and grading of rice grains. Features like length of seed, width, and perimeter are specially considered.

From the literature survey it is evident that major work is done on identification of different types of food grains but very less amount of work has been reported on the grading of rice. Hence it is important that a automated process is essential to replace manual process of grading rice is required to be replaced with "Digital image processing technique".

\section{What is seed?}

A seed is a small embryonic plant enclosed in a covering called the seed coat, usually with some stored food.

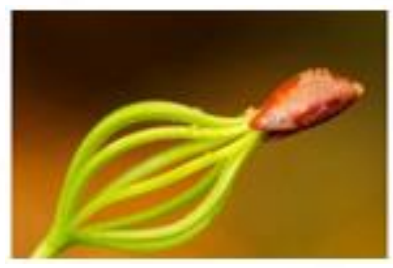

Fig 3: Seed

\section{WHY QUALITY OF SEED IS MPORTANT?}

It is very important to use quality seed in rice production. Using quality seed can increase your yield between 5 and 20 percent. Using seed of lesser quality will introduce more weeds and off-types into your crop, make the crop more susceptible to disease, and produce plants that are weaker. By using quality seed, you will save money on the inputs to your crop and, because of higher yields; you will get more income or have more food for the family.

\section{WHY IMAGE PROCESSING ECHNIQUES?}

Rice is one of the most important grain crops in India. The quality of rice seeds has major effect on the yield 
of rice, so the proper inspection of rice seed quality is very much important now days. The purity of seed is one of the factors whose inspection is more difficult and more complicated than that of other factors.

Presently, the identification of rice seed variety mainly depends on chemical method and paddy field method. These two methods can give more exact results but they have many limitations. Application of the chemical method is very high expense for inspection. The inspection of seed using the paddy field method is too long to satisfy the demand of seed circulation. The nondestructive identification of rice seed variety on a large scale cannot be achieved by using these two methods. So non destructive, nonexpensive inspection using machine vision based on digital image processing technology is much faster.

\section{PROPOSED METHODOLOGY}

In the proposed method we have considered three varieties of rice grains namely Basmati, Basmati mogra and Basmati Tukda. Each of these grains are further classified and graded into grade 1, grade 2 and grade 3. In the Manual process grading is done manually. Based on the features like major axis length, minor axis length, area, eccentricity and perimeter experts grade the grains. The same features are used in automated method for grading of the grains. Fig.4 shows Grading of rice grains.

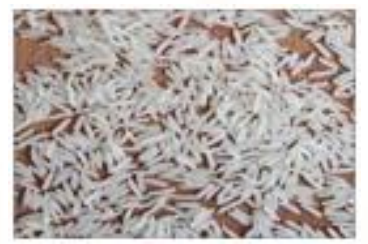

Grade 1

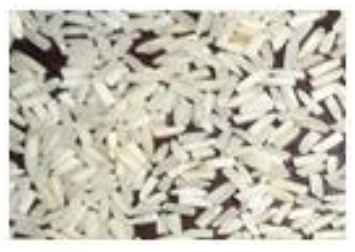

Grade 2

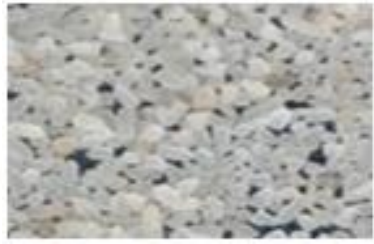

Grade 3
Fig 4: Grading of rice grains manually Classified

\section{BLOCK DIAGRAM}

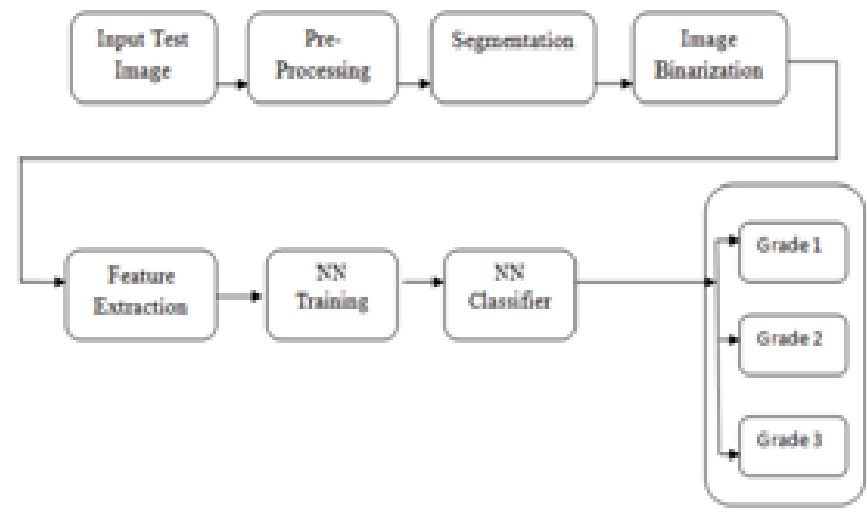

Fig 5: Block diagram of the grading system

Figure 5 shows the actual block diagram of the grading system.Figure 6 shows the circuit diagram used for the LCD for showing results of different rice grades.

\section{CIRCUIT DIAGRAM}

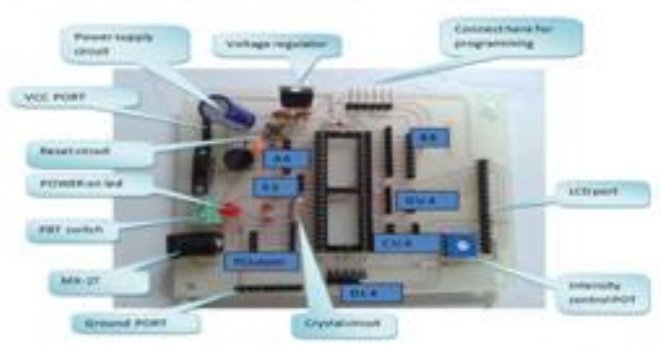

Fig 6: Circuit diagram of the LCD

\section{ALGORITHM}

$>$ Pre-process the images of grain to remove background noise

$>$ Convert the preprocessed image to binary image using Otsu method.

$>$ Region label the binary image.

$>$ Segment/crop the individual grains present in the image.

$>$ Extract the geometric features major axis, minor axis ,essentricity,perimeter and area of all the individual grains

$>$ Train Artificial Neural Network

$>$ Use ANN Classifier to Divide image into different quality Levels

Stop

IX. CRITERIA USED FOR GRADING 
Images are acquired using INTEX high resolution camera. The rice samples are placed on the sheet of the paper and the images were captured. The camera was placed at a position normal to the rice samples. The images were stored in JPG format. A data set of different set of images was created manually classified Shown in fig. Fig 4. Fig.7 shows the flow diagram for grading of rice grains, the steps involved here are input image, pre processing, grain extraction, counting, analysis and results.

The work focused quality analysis on the basis of the measurement of physical parameter i.e. grain size and shape using image processing techniques. Basmati rice is used for quality analysis. Basmati rice is classified as extra long, long, medium and short. The image processing technique is used for counting the number of rice seeds and classifies them on the basis of length, breadth and length breadth ratio. Length is the average length of rice grain while breadth is the average breadth of rice grain and length-breadth ratio is calculated as;

$\mathrm{L} / \mathrm{B}=[($ Avg. length of rice) $/$ (Avg. breadth of rice) $]^{*} 100$

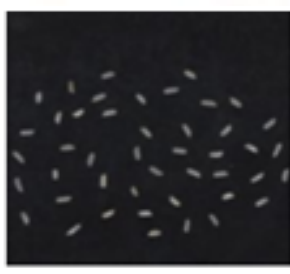

(a)

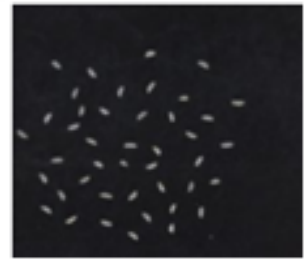

(b)

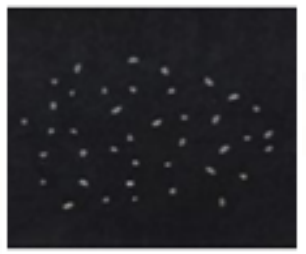

(c)

Fig 7 shows grading of rice grains (a) Sample image of Basamati grade1 (b) Sample image of grade2 and (c) Sample Image of grade3 rice. .

The above fig. 7 shows three qualities of rice grains we consider for grading, here we have considered Basamati rice , Basamati Mogra and Basmati Tukda for grading. Figure 7 (a), (b) and (c) shows the rice grains graded as grade 1 , grade 2 and grade 3 and result will be displayed on LCD. Testing Results for Quality analysis and grading of rice is shown in the below table 1 .

\section{X . RESULS AND MEASUREMENTS}
Table 1: Testing Results for quality analysis and grading of rice

\begin{tabular}{|c|c|c|c|}
\hline $\begin{array}{c}\text { Rice } \\
\text { Grade }\end{array}$ & $\begin{array}{c}\text { Number } \\
\text { of } \\
\text { samples }\end{array}$ & $\begin{array}{c}\text { Classification } \\
\text { Result }\end{array}$ & $\begin{array}{c}\text { Accuracy } \\
(\%)\end{array}$ \\
\hline Grade 1 & 20 & $\begin{array}{c}18 \text { are classified } \\
\text { as grade 1 }\end{array}$ & 98 \\
\hline Grade 2 & 20 & $\begin{array}{c}19 \text { are classified } \\
\text { as grade 2 }\end{array}$ & 99 \\
\hline Grade 3 & 20 & $\begin{array}{c}19 \text { are classified } \\
\text { as grade 3 }\end{array}$ & 99 \\
\hline
\end{tabular}

Fig 8: Camera used for grading

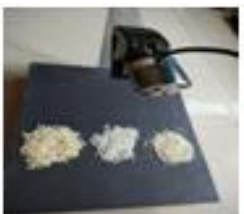

Fig 9: Actual photos of System

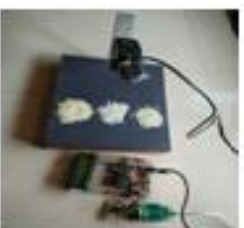

Fig 10: Actual photos of System with LCD

\section{CONCLUSION}

Now days consumers are very quality conscious about the food grains they buy and consume. In this paper an attempt is made to grading of rice grains based on morphological techniques using image processing and Artificial Neural Network. The image is initially subjected to preprocessing and the individual grains are segmented by using different image processing techniques. The geometric features of the grain such as area, major axis length, minor axis length ,eccentricity and perimeter of rice seeds are extracted and are subjected to classification The result are found to be encouraging. Based on the length of the grain 
the rice is graded as grade 1 , grade 2 and grade 3 . Neural Network Pattern Recognition Tool is applied in grading rice granules. The developed Neural Network can be adapted for grading added grains and foodstuffs as well. Scaled Conjugate Gradient Training based Neural Network is able to classify well when there is no overlapping of granules with the accuracy of $98.7 \%$. When overlapping of granules is available then the accuracy will be $90 \%$. The average accuracy of classification is found to be $98 \%$. Result will be displayed on LCD and also there is an audio message.

\section{Acknowledgment}

We sincerely thankful to all teaching staff as well as ME students for their kind support. We thankful to HOD E\&TC, Principal and campus director of JSPM's JSCOE, for providing us all necessary facilities.

\section{REFERENCES}

[1] Bhagyashree Mahale, Prof. Sapana Korde, Rice Quality Analysis Using Image Processing. International Conference for Convergence of Technology - $2014 \quad 978-1-4799-3759-$ 2/14/\$31.00@2014 IEEE

[2] Gurpreet Kaur \& Bhupinder Verma, Measurement standards based grading of rice kernels by separating touching kernels for embedded imaging applications, International Journal of Electronics, Communication \& Instrumentation Engineering Research and Development (IJECIERD) ISSN 2249-684X, Vol. 3, Issue 1, pp 127-134, Mar 2013.

[3] Jagdeep Singh Aulakh, Dr. V.K. Banga. Grading of rice grains by image processing. International Journal of Engineering Research \& Technology (IJERT) Vol. 1 Issue 4, pp 1-4,June 2012

[4] Chetna V. Maheshwari,[Q-CURVE APPROACH FOR QUALITY ANALYSIS OF INDIAN ORYZA SATIVA SSP INDICA(RICE), International Journal of Advanced Technology in Engineering and Science, Volume No.01, Issue No. 03, March 2013.

[5] Harpreet Kaur, Baljit Singh, ijsrp, [5] classification and grading of rice grains using multi-class SVM. Volume 3, Issue 4 April 2013.

[6] Megha R. Siddagangappa A. H. Kulkarni,[6] Classification and Quality Analysis of Food
Grains. IOSR-JCE, Volume 16, Issue 4, Ver. III, pp 01-10 jul-aug 2014.

[7] G.Ajay, M.Suneel,Quality evalution of rice grains using morphological methods, International Journal of Soft Computing and Engineering (IJSCE) ISSN: 2231-2307, Volume-2, Issue-6, pp 35-37, January 2013.

[8] Basavaraj S. Anami, Dayanand G. Savakar,[5] Effect of Foreign Bodies on Recognition and Classification of Bulk Food Grains Image Samples. Journal of Applied Computer Science, no. 6 (3), pp 77-83 2009. 